\title{
PROKLA-Redaktion
}

\section{Editorial: Geopolitische Konflikte nach der „neuen Weltordnung"}

Nur ein knappes Vierteljahrhundert nach ihrer Ausrufung durch den damaligen USPräsidenten George H.W. Bush befindet sich die „neue Weltordnung " in Auflösung. Der Konflikt in der Ukraine, die (Bürger-) Kriege im Nahen Osten und der Aufstieg des IS, die jüngsten Fluchtbewegungen, die Weltwirtschaftskrise und die durch sie beschleunigten Verschiebungen zugunsten der BRICS-Staaten (Brasilien, Russland, Indien, China, Südafrika), die Krise in der EU, die Konflikte um natürliche Ressourcen und nicht zuletzt ökologische Krisenphänomene wie der Klimawandel haben die Vorstellung einer friedlichen globalen Entwicklung unter kapitalistischen Vorzeichen und unter US-amerikanischer Führung gründlich desavouiert.

Was an ihre Stelle treten könnte, ist noch unklar, die Konturen des Neuen zeichnen sich erst verschwommen ab. $\mathrm{Zu}$ bestätigen scheint sich allerdings, dass das Neue von jener „Vereinheitlichung und Fraktionierung" geprägt sein wird, die Michael Bonder, Bernd Röttger und Gilbert Ziebura schon 1993 in der PROKLA 91 diagnostiziert haben. Einerseits verschärfen sich im Zuge der jüngsten Weltwirtschaftskrise die Klassenkämpfe und überlagern sich mit Territorialkonflikten (Ostasien, die Ukraine oder der arabische Raum). Andererseits werden Projekte wie das Transatlantische Freihandelsabkommen (TTIP) und das internationale Dienstleistungsabkommen TISA vorangetrieben, die als Versuche einer institutionellen Vereinheitlichung aber ihrerseits die soziale und regionale Fragmentierung in den beteiligten Ländern vertiefen dürften.

Davon bleiben auch die wissenschaftlichen Beschreibungen der "neuen Weltordnung" nicht unberührt. Die in den 1990er Jahren vorherrschenden Konzepte wie Global Governance oder Multilateralismus waren von Beginn an eher normativ überfrachtet als analytisch gesättigt. Wohlwollend begleiteten sie die Versuche der sogenannten Staatengemeinschaft, nach dem Ende des Ost-West-Konflikts globale Probleme wie den Klimawandel, das Entwicklungsgefälle zwischen Erster und Dritter Welt oder die in vielen Ländern zunehmenden innergesellschaftlichen Spaltungen kooperativ zu bearbeiten. In einer Serie von großen UN-Konferenzen beginnend mit der Konferenz über Umwelt und Entwicklung 1992 in Rio der Janeiro und endend mit der Habitat-Konferenz über menschliche Siedlungen 1996 in Istanbul - schienen sie ihre empirische Bestätigung zu finden.

Was durch die Multilateralismus- und Global-Governance-Brille kaum wahrgenommen wurde, war, dass es auch auf dem scheinbar neutralen Terrain der UN-Verhandlungen nicht in erster Linie um „problem-solving“, sondern um Interessenpolitik ging. Zudem blieb unterbelichtet, dass die umwelt- und entwicklungspolitischen „Menschheitsfragen“ 
immer schon im „Schatten der Hierarchie“ (Fritz Scharpf) verhandelt wurden, wie er von den Organisationen der neoliberalen Globalisierung geworfen wurde - dem Internationalen Währungsfonds (IWF) und der Welthandelsorganisation (WTO) bzw. ihrem Vorläufer, dem Allgemeinen Zoll- und Handelsabkommens (GATT). Dass das Scheitern der "kooperativen“ Problembearbeitung bereits in dem „Rio-Typus von Politik" der 1990er Jahre (Ulrich Brand/Christoph Görg) selbst angelegt war, musste den Multilateralismus- und Global-Governance-Konzepten deshalb zwangsläufig entgehen.

Aber auch das Empire-Konzept, mit dem Michael Hardt und Antonio Negri im Jahr 2000 gleichsam einen linken Gegenentwurf zum Idealismus des Mainstreams vorlegten, hatte nur eine kurze Halbwertzeit. Wohl hat sich die von ihnen diagnostizierte „reelle Subsumtion der Gesellschaft unter das Kapital", also die kapitalistische Inwertsetzung von immer mehr gesellschaftlichen Bereichen, in jüngerer Zeit infolge von Krise und Austeritätspolitik eher noch intensiviert. Die These aber, dass dem Kapitalismus auch im internationalen Maßstab das Außen verloren gegangen und folglich an die Stelle imperialistischer Konkurrenz ein weltumspannendes, netzwerkförmiges Herrschaftsverhältnis getreten sei, in dem die USA und internationale Organisationen wie die WTO oder der IWF die zentrale Rolle spielen, lässt sich angesichts der zunehmenden geopolitischen und -ökonomischen Spannungen sowie der Krise der WTO kaum mehr aufrechterhalten.

Einer Überprüfung bedarf des Weiteren das American-Empire-Konzept von Leo Panitch und Sam Gindin. Im Unterschied zu Hardt und Negri gingen sie zwar nicht von der Herausbildung eines weltumspannenden Empire aus. Ähnlich wie jene vertraten sie jedoch die These, dass die territoriale Konkurrenz nicht mehr das strukturierende Prinzip der Weltordnung darstelle. Stattdessen seien die kapitalistischen Zentren unter Führung der USA in wirtschaftlicher, politischer, militärischer und kultureller Hinsicht eng miteinander verflochten. Die entscheidende Herausforderung dieses American Empire sei es, die Beziehungen zu seinem Außen, vor allem zum globalen Süden, zu regulieren. Es ist keineswegs ausgemacht, ob dies angesichts der ökonomischen, politischen und militärischen Herausforderungen, mit denen die kapitalistischen Zentren heute konfrontiert sind und mit denen sich die Spannungen zwischen ihnen vergrößern, so noch gilt.

Am ehesten noch scheint die Debatte über einen "neuen Imperialismus", die in den frühen 2000er Jahren einsetzte, diesen Herausforderungen begrifflich gewachsen zu sein. Ihr wohl bekanntester Vertreter, David Harvey, schätzte die Möglichkeiten der USA, die propagierte „neue Weltordnung" tatsächlich in ihrem eigenen Sinne zu gestalten, von vornherein skeptischer ein als Hardt und Negri oder Panitch und Gindin. Angeregt durch die Weltsystemtheorie Giovanni Arrighis ging er davon aus, dass den USA in Gestalt von China ein ernstzunehmender ökonomischer Konkurrent erwachsen sei, mit dessen Aufstieg sich das weltwirtschaftliche Gravitationszentrum vom atlantischen Raum nach Ostasien verschieben könnte. Was den USA jedoch bleibe, sei ihre militärische Überlegenheit, die sie zur Kompensation ihres relativen politischen und ökonomischen Bedeutungsverlusts zu nutzen versuche. In diesem Sinne interpretierte Harvey auch den Irak-Krieg von 2003: als Versuch der USA, mittels einer Kontrolle über die globalen Ölvorräte bzw. über die Bedingungen, unter denen diese gefördert und verteilt werden, aufdas ressourcenintensive Wachstumsmodell Chinas einzuwirken. 
In der PROKLA wurden diese Entwicklungen und Debatten immer wieder aufgegriffen. So spielten sie eine Rolle in Beiträgen zur kriegerischen Intervention in Libyen (PROKLA 163/2011) oder zur US-Außenpolitik unter Obama. Der Beitrag „Weltpolizist Obama“ von Horst Heitmann in der PROKLA 169/2012 kann dabei als Selbstkritik gelesen werden, denn auch in der PROKLA (siehe Heft 153/2008) wurde der Regierungswechsel von Bush zu Obama positiver eingeschätzt, als er dann tatsächlich war. In Beiträgen zur Weltsystemtheorie oder zu Imperialismus war Geopolitik de facto immer ein Thema. Dabei spielte der Niedergang der US-Hegemonie eine wichtige Rolle (siehe PROKLA 153/2008: Die USA nach Bush). In den 1980er Jahren wurde diese im Unterschied zu späteren Zeitpunkten (siehe PROKLA 119/2000 und PROKLA 161/2010) nicht im Kontext des Aufstiegs Chinas analysiert. Vielmehr galt Japan als das ökonomische Schwergewicht, das den globalen Kapitalismus zu verändern schien (PROKLA 66/1987).

Auffallend ist, dass vor 1990 zwar der US-Imperialismus ein viel diskutiertes Thema war, dass aber die geopolitische Gesamtsituation aufgrund des sogenannten Kalten Kriegs als stabil erschien und nur wenig thematisiert wurde. Das änderte sich 1991, als in der PROKLA 84 die „Neuaufteilung der Welt“ diskutiert wurde. Es folgten Hefte zur imperialistischen Globalisierung (PROKLA 133/2003) und zu Ressourcenkonflikten (PROKLA 135/2004), zur Internationalisierung des Staates (PROKLA 147/2007) und zu Krieg und Frieden (PROKLA 162/2011). Das vorliegende Heft knüpft an diese Schwerpunkte an und bemüht sich um eine Einordnung der vielfältigen Konflikte, die die Zeit „nach der neuen Weltordnung“ prägen. Die Konflikte waren und sind nicht immer „kriegerisch“, was uns dazu bewog, das vorliegende Heft nicht unter der Überschrift „Imperialismus“, sondern „Geopolitik“ zu veröffentlichen.

Im ersten Beitrag lotet David Salomon den Erkenntniswert der jüngeren und älteren Imperialismus-Debatte aus. Beginnend mit den klassischen (marxistischen) Arbeiten gibt er einen Überblick über die Entwicklung der theoretischen Auseinandersetzung mit dem Imperialismus bis in die heutige Zeit. Der Schwerpunkt seiner Betrachtungen liegt dabei auf den Jahrzehnten seit Ausrufung der „neuen Weltordnung“. Sei zunächst ein "guter“, aufKooperation und die weltweite Verbreitung von Marktwirtschaft und liberaler Demokratie hin orientierter Imperialismus vorherrschend gewesen, so habe dieser sich nach den Anschlägen vom 11. September $2001 \mathrm{zu}$ einem stärker militärisch geprägten gewandelt. Heute, so Salomons zentrale These, haben wir es mit einer multipolaren „Ordnung rivalisierender Imperien“ zu tun, in der die USA zwar nach wie vor den ersten Rang einnehmen, jedoch immer stärker mit alten Verbündeten wie der EU und neuen Mächten wie den BRICS-Staaten sowie mit den Allianzen, die letztere untereinander schließen, konkurrieren.

Vor allem die geopolitische Konkurrenz zwischen den USA und Russland hat mit der russischen Annexion der Krim im März 2014 sowie mit der militärischen Unterstützung des syrischen Diktators Assad durch Russland neue Nahrung erhalten. Felix Jaitner untersucht in seinem Beitrag die gesellschaftlichen Kräfteverhältnisse, die der „außenpolitischen Renaissance“ Russlands zugrunde liegen. Sein Ausgangspunkt ist der in den 1990er Jahren eingeschlagene, auf der Extraktion von natürlichen Ressourcen beruhende und von Oligarchen dominierte periphere Entwicklungsweg Russlands. Dieser wurde unter Putin zugunsten einer 
stärker auf die Reindustrialisierung des Landes setzenden und von einem Kompromiss zwischen Staatsbürokratie und Oligarchen getragenen Strategie, wenn nicht verlassen, so doch korrigiert. Eine stärkere Anbindung der Ukraine, die vor allem im Osten über ein großes industrielles Potenzial verfügt, ist, so Jaitner, Teil dieser veränderten russischen Strategie, während das militärische Eingreifen in Syrien eher einer politischen Destabilisierung an den Rändern des russischen Einflussbereichs entgegenwirken soll.

Malte Daniljuk analysiert in seinem Beitrag die US-Energiepolitik als Medium einer geopolitischen und -ökonomischen Neuordnung. Er zeichnet die Bestrebungen der USA nach, durch die Förderung ihrer einheimischen, nicht-konventionellen Öl- und Gasproduktion zur „Energiesupermacht" aufzusteigen. Die jüngst drastisch gefallenen Preise für Öl und Gas sind nicht zuletzt dieser Strategie geschuldet. Ihre Wirkung ist jedoch widersprüchlich: Einerseits untergräbt der Erfolg der USEnergiepolitik ihre eigenen Grundlagen, denn die Rentabilität der nicht-konventionellen Öl- und Gasproduktion hängt von hohen Energiepreisen ab. Andererseits wirken die niedrigen Energiepreise wie ein "gigantisches Konjunkturprogramm "und unterstützen die USA in ihrem Bemühen, sich als zentrales Glied einer Kette von Freihandelsabkommen - im Osten mit Asien, im Süden mit Lateinamerika und im Westen mit Europa - zu etablieren.

Stefan Schmalz diskutiert die geopolitischen Folgen des chinesischen Aufstiegs, der zu einer Vielzahl neuer struktureller Veränderungen führt. Er untersucht, wie weit Chinas globales Engagement heute fortgeschritten ist und ob es eine Herausforderung für die USA und ihre europäischen Verbündeten darstellt. Die Volksrepublik China sei dabei, ihre wachsende wirtschaftliche Bedeutung auch in strukturelle Macht im globalen Kapitalismus umzusetzen und bringe damit auch das American Empire an seine Grenzen.

Zwei weitere Beiträge widmen sich den geopolitischen Ambitionen der EU, einzelner europäischer Kapitalfraktionen bzw. Deutschlands als mächtigstem EUMitgliedstaat. In der PROKLA 180/2015 hat Michael Heinrich die These vertreten, dass die Europäische Wirtschafts- und Währungsunion entgegen der Annahme ihrer linkskeynesianischen KritikerInnen keineswegs eine austeritätspolitische Fehlkonstruktion sei, die sich früher oder später auch gegen das deutsche neo-merkantilistische Modell wenden würden. Stattdessen sei die Austeritätspolitik gewollt und im Interesse Deutschlands. Frederic Heine und Thomas Sablowski untermauern diese These nun mit empirischen Befunden. Sie zeigen, dass die EU, allen voran die krisengeschüttelten Länder im Süden, für Deutschland als Absatzmarkt an Bedeutung verloren, der Weltmarkt dagegen an Bedeutung gewonnen hat. Vor diesem Hintergrund seien die Krise in der EU und die damit verbundene Schwächung des Euro für Deutschland keineswegs dysfunktional. Vielmehr stärkten sie die Wettbewerbsfähigkeit der weltmarktorientierten Kapitalfraktionen in Deutschland.

Christina Plank untersucht die ökonomischen Interessen der EU an der Ukraine. Sie stellt fest, dass europäische Unternehmen vor allem in die Landwirtschaft, die Energieversorgung und den Banken-Sektor der Ukraine investiert haben. Allerdings stießen sich ihre Interessen immer wieder an den von den ukrainischen Oligarchen geprägten gesellschaftlichen Strukturen. Als nationale Bourgeoisie widersetzten sich die Oligarchen einer Internationalisierung der ukrainischen Wirtschaft. Die europäischen Kapitalstrategien allein erklärten deshalb nicht die Eskalation des 
Ukraine-Konflikts seit 2014. Vielmehr sei es nötig, im Anschluss an Giovanni Arrighi und David Harvey zwischen einer kapitalistischen und einer geopolitisch-territorialen Logik der Macht zu unterscheiden. Im Fall der Ukraine, so Plank, hat letztere sich gegenüber ersterer verselbständigt und zur Intensivierung des Konflikts geführt.

Wolfram Schaffar befasst sich mit dem Hochgeschwindigkeitsbahn-Projekt One Belt, One Road, mit dem China die infrastrukturellen Voraussetzungen für die regionale ökonomische Integration $\mathrm{zu}$ verbessern versucht. Das Projekt lasse sich als Ausdruck des Aufstiegs Chinas und des damit einher gehenden „Umbruchs zu einem neuen Akkumulationszyklus der Weltwirtschaft" begreifen. Am Beispiel Thailands untersucht Schaffar die gesellschaftlichen und politischen Verwerfungen, die dieser Umbruch an der Peripherie der neuen Großmacht erzeugt. Er interpretiert die Kämpfe zwischen Gelb- und Rothemden als Konflikt gesellschaftlicher Kräfte um die Teilhabe an dem Übergang in eine neue, von China dominierte hegemoniale Konstellation. Die Tatsache, dass China selbst sich diesem Konflikt gegenüber indifferent verhält, ermögliche einen Einblick in den spezifischen Charakter seiner Hegemonialpolitik: Im Unterschied zu den USA, die ihre Hegemonie auf Verträge und das politische Wohlverhalten ihrer Partner gründeten, setze China stärker auf eine infrastrukturelle Anbindung, die Raum für unterschiedliche politische Optionen lasse.

Außerhalb des Schwerpunkts setzt sich Sybille Bauriedl kritisch mit der internationalen Klimapolitik auseinander. Anlässlich der gerade beendeten 21. Vertragsstaatenkonferenz der UNKlimarahmenkonvention in Paris geht sie der Frage nach, warum die dramatischen Problembeschreibungen der Klimawissenschaft bislang keine wirksame
Klimapolitik nach sich gezogen haben. Bauriedl zufolge sind die wissenschaftlichen Beschreibungen des Klimawandels selbst Teil des Problems: In Konzepten wie "Anthropozän“ oder "planetarische Grenzen“würden die gesellschaftlichen Macht- und Herrschaftsverhältnisse, die dem Klimawandel zugrunde liegen und die für die höchst ungleiche Verteilung seiner Folgen verantwortlich sind, vernachlässigt. Statt globale und soziale Ungleichheiten zu problematisieren, suggerierten die in der Klimawissenschaft vorherrschenden Konzepte eine gemeinsame, Staaten- und innergesellschaftliche Grenzen überschreitende Verantwortung, der mit Hilfe von technologischen und marktförmigen Mitteln Rechnung getragen werden soll.

Vor dem Hintergrund der jüngeren Entwicklungen in Griechenland und Spanien eröffnet Dario Azzelini eine Debatte über die Möglichkeiten und Grenzen, über linke Parteipolitik in den bestehenden Institutionen eine grundlegende gesellschaftliche Transformation anzustoßen. Syriza stehe für ein Scheitern dieser Strategie. Die Partei habe es versäumt, sich stärker auf eine gesellschaftliche und institutionell möglicherweise nicht mehr kanalisierbare Protestdynamik einzulassen. Auch Podemos drohe sich von den sozialen Bewegungen abzukoppeln, um für eine Mehrheit wählbar zu sein. Die katalanische CUP stehe dagegen für einen parteipolitischen Ansatz, der die Beteiligung an sozialen Kämpfen mit institutioneller Politik zu verbinden versucht. Indem sie innerhalb der Institutionen gegen die konstitutionelle Ordnung des spanischen Staates arbeitet, könne sie eine Dynamik auslösen, die „große Chancen sozialer Transformation" beinhalte. Die Redaktion lädt zu weiteren Debattenbeiträgen ein.

Ingo Stützle und Markus Wissen (für die Redaktion) 